\title{
Sexual Function in Cancer Survivors: Updates to the NCCN Guidelines for Survivorship
}

\author{
Presented by Michelle E. Melisko, MD, and Joseph B. Narus, DNP, APRN, NP
}

\begin{abstract}
By the year 2024, predictions estimate that there will be approximately 19 million cancer survivors in the United States, many of whom will experience the physical and/or psychosocial long-term effects of cancer and its treatment. Although sexual dysfunction is common among these individuals, causing increased distress and negatively impacting quality of life, this critical part of survivorship care is often underrecognized and undertreated. At the NCCN 21st Annual Conference, Drs. Melisko and Narus reviewed the sexual function recommendations in the NCCN Guidelines for Survivorship, offering practical strategies for assessing and managing both female and male sexual dysfunction. J Natl Compr Canc Netw 2016;14(5.5):685-689
\end{abstract}

As a breast cancer specialist, I did not really know how common these problems [sexual dysfunction] were in other areas of cancer," revealed Michelle E. Melisko, MD, Associate Clinical Professor of Hematology/Oncology, Department of Medicine, University of California San Francisco Helen Diller Family Comprehensive Cancer Center. "Three of the 4 top cancer sites in men involve the pelvic region," often affecting male sexual function, concurred Joseph B. Narus, DNP, APRN, NP, a nurse practitioner in the Male Sexual and Reproductive Medicine Program, Memorial Sloan Kettering Cancer Center, and the first nurse practitioner to manage the Penile Rehabilitation Program.

\footnotetext{
Presented by Michelle E. Melisko, MD, Department of Medicine, Division of Hematology/Oncology, Helen Diller Family Comprehensive Cancer Center, University of California San Francisco, San Francisco, California, and Joseph B. Narus, DNP, APRN, NP, Male Sexual and Reproductive Medicine Program, Urology Service, Memorial Sloan Kettering Cancer Center, New York, New York.

Dr. Melisko has disclosed that she receives consulting fees and/or honoraria from Agendia BV and Nektar Therapeutics, and grant/ research support from Eli Lilly and Company, Galena Biopharma, and Genentech, Inc.; and that her spouse/partner has equity interest/stock options in Merrimack Pharmaceuticals, and receives consulting fees and/ or honoraria for Pfizer Inc. and Genentech, Inc. Dr. Narus has disclosed that he has no financial interests, arrangements, affiliations, or commercial interests with the manufacturers of any products discussed in this article or their competitors.

Correspondence: Michelle E. Melisko, MD, Department of Medicine, Division of Hematology/Oncology, Helen Diller Family Comprehensive Cancer Center, University of California San Francisco, 1600 Divisadero Street, $2^{\text {nd }}$ Floor, San Francisco, CA 94115. E-mail: mmelisko@medicine.ucsf.edu

Joseph B. Narus, DNP, APRN, NP, Male Sexual and Reproductive Medicine Program, Urology Service, Memorial Sloan Kettering Cancer Center, 1275 York Avenue, New York, NY 10065.

E-mail: narusj@mskcc.org
}

\section{Scope of the Problem}

For many patients undergoing treatment for cancer, surgery, chemotherapy, and endocrine therapy may cause side effects during and after treatment, with sexual dysfunction among them. For instance, oophorectomy causes acute estrogen deprivation, hysterectomy may change vaginal length and size, prostate surgery can cause erectile dysfunction and impotence, and breast and colorectal surgeries may impact body image and self-esteem, explained Dr. Melisko, who also is a member of the NCCN Survivorship Panel.

"Chemotherapy can affect sexual function in some not so obvious ways," stated Dr. Melisko. For instance, adverse effects of treatment may include reduced sexual desire, decreased libido, and fatigue. In addition, "Endocrine therapy has a profound impact on both men and women," she added. Along with the effects of estrogen deprivation (eg, vasomotor symptoms, sleep disturbance, vaginal dryness), mood changes can result in decreased libido.

Dr. Melisko briefly illustrated the impact of cancer and its treatment on the sexuality of survivors of several different types of cancer. For instance, female survivors of cervical and breast cancers (and their treatments) have experienced persistent and substantial sexual morbidity, often for months to years after treatment. ${ }^{1,2}$ In fact, the long-term use of hormonal agents such as tamoxifen and aromatase inhibitors can cause vasomotor and musculoskeletal symptoms. ${ }^{3}$ For both men and women who have had surgery for rectal cancer, sexual 
problems afterward are "common, multifactorial, inadequately discussed, and untreated," according to the literature. ${ }^{4}$

\section{Steps in Diagnostic Evaluation}

The first step in the diagnostic evaluation for sexual dysfunction is to ask patients about it at regular intervals. In the 2016 NCCN Guidelines for Survivorship, the recommendations regarding the management of sexual dysfunction include sexual function checklists for screening both men and women. Among the validated screening instruments is the Brief Sexual Symptom Checklist for women. The final question asks whether the patient would like to talk about sexual function with a health care professional.

The Sexual Health Inventory for Men (SHIM) is a short checklist for evaluating erectile dysfunction. The 5 questions address sexual activity over the past 6 months, with satisfaction/dissatisfaction with erectile function and sexual intercourse being the main topics. Clinical experience with this widely used scale has indicated "its positive impact on understanding and improving male sexual function." 5

Another similar screening tool for men is the International Index of Erectile Function (IIEF), a questionnaire covering 5 domains: erectile function, orgasmic function, sexual desire, intercourse satisfaction, and overall satisfaction. ${ }^{6}$ The main difference between the 2 male screening tools is that SHIM refers to sexual activity in the past 6 months, whereas IIEF refers to sexual activity in the past 4 weeks, explained Dr. Narus.

Answers to these screening questions guide subsequent steps in the 2016 NCCN Guidelines. For survivors of both sexes who indicate sexual concerns - and want to discuss them further-oncologic history should be reviewed. Patients' medical history should also be reviewed for conditions that may be associated with sexual dysfunction (eg, depression, diabetes, hypertension). Dr. Narus noted that certain medications, such as selective serotonin reuptake inhibitors and beta-blockers; depression; and anxiety can also impact male sexual health in men treated for cancer.

However, assessment for sexual dysfunction in men includes more than just medical and surgical histories, use of validated screening tools, physical examination, and laboratory tests, Dr. Narus added.
A sexual history should be obtained as well. "When we speak to our patients, we have to respect their privacy and also be comfortable opening up about other issues," Dr. Narus continued.

\section{Treatment Alternatives for Women}

The 2016 NCCN Survivorship Guidelines for Sexual Function contain a new algorithm for assessing women with concerns or issues regarding sexual function. Appropriate treatment options and follow-up recommendations are based on the types of patientreported symptoms (Figure 1). The first category of symptoms focuses on those relating to menopause, vaginal dryness, or other issues related to vaginal health (eg, discomfort, discharge, pain).

Dr. Melisko briefly reviewed the past findings of the Women's Health Initiative in regard to the management of vaginal atrophy and menopausal symptoms in women with nonhormonally sensitive cancer. Hormone-replacement therapy is no longer indicated as a result of these findings and because of the increased risks of breast cancer, heart attack, and stroke. However, admitted Dr. Melisko, a closer look at these study data suggested that there might be some benefit to estrogen use in younger women (aged $<60$ years).

Limited data are available from randomized controlled trials in women with breast cancer regarding the safety of drugs for treating urogenital atrophy, Dr. Melisko said. For example, a recent review of the sparse data indicated that although urogenital atrophy may be common in these survivors, "optimal management is currently unknown, and treatment remains a challenging issue" —-one that requires more prospective trials.

Dr. Melisko offered a glimpse at some of the other treatment options listed in the 2016 NCCN Guidelines for Survivorship, including vaginal moisturizers, vaginal gels, oils, topical vitamin $\mathrm{D}$ or $\mathrm{E}$, and lubricants for sexual activity. However, she admitted, there is a lack of clear-cut data in support of one of these options over another, particularly in breast cancer survivors.

Topical prescriptions and the nonestrogen selective estrogen receptor modulator ospemifene are listed as treatment options as well, although FDAapproved ospemifene is contraindicated in survivors with a history of estrogen-dependent cancers. This agent is indicated for the treatment of vaginal dryness in postmenopausal women with vulvovaginal 


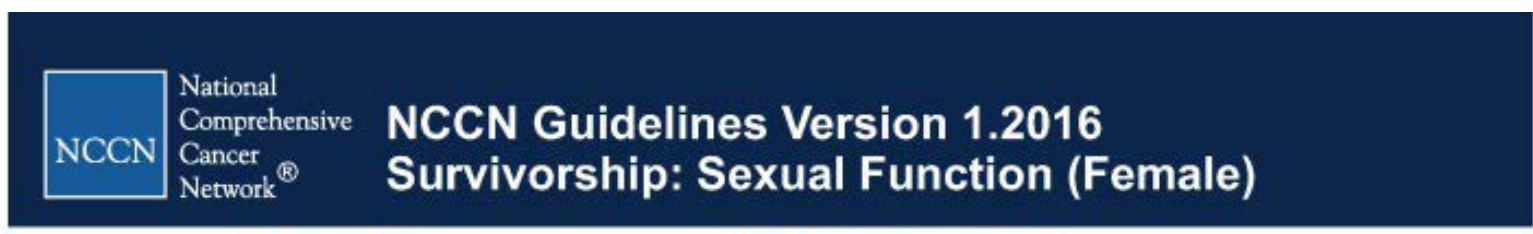

SYMPTOMS

TREATMENT OPTIONS

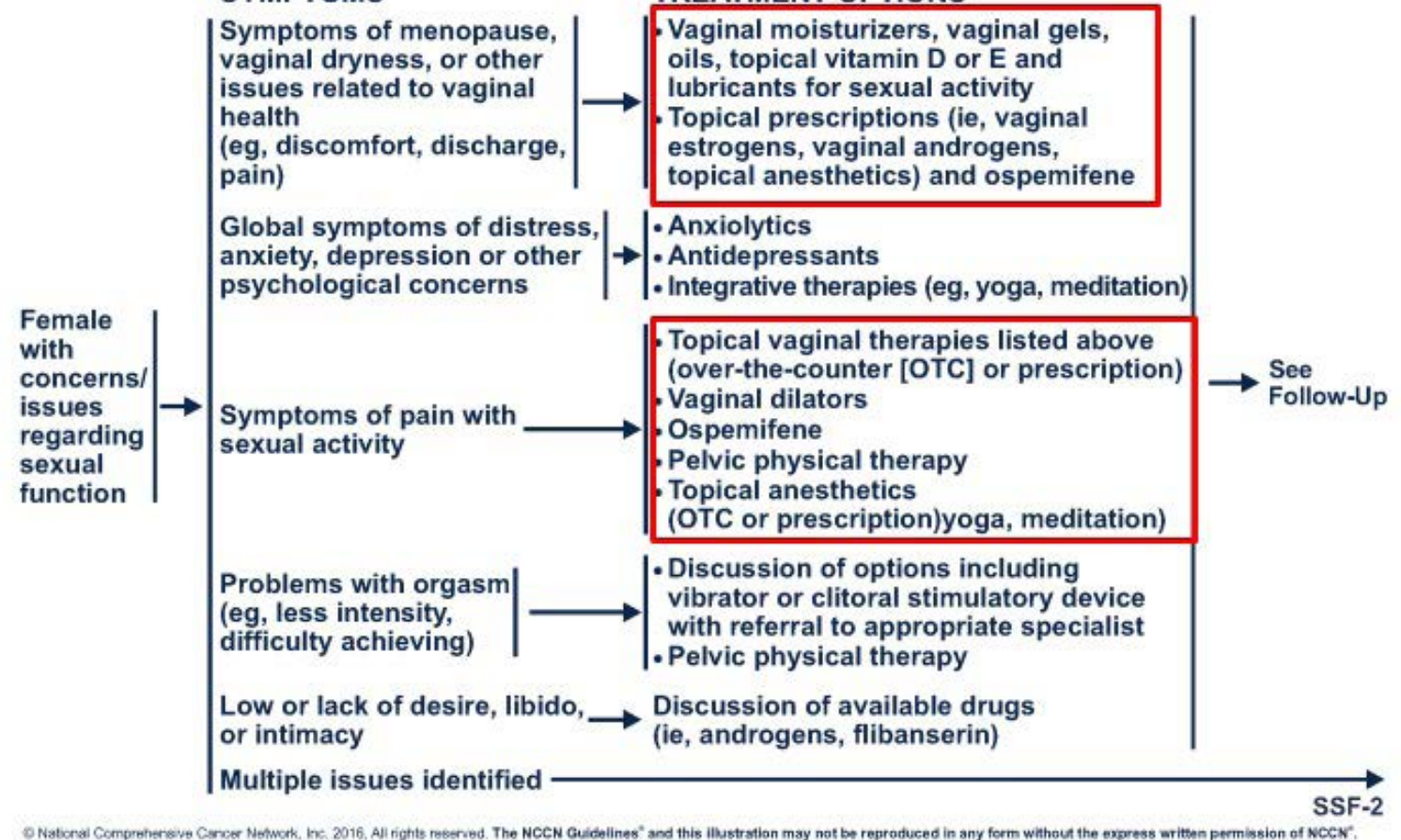

Figure 1 NCCN Survivorship Guidelines recommendations/treatment options for females with sexual dysfunction.

atrophy, ${ }^{8}$ but it is not approved for use in breast cancer survivors, revealed Dr. Melisko; it may, however, be useful for survivors of other cancers, she added.

For symptoms of dyspareunia, treatment options include topical vaginal therapies, vaginal dilators, topical anesthetics, and ospemifene. Dr. Melisko focused attention on a recent randomized controlled trial of liquid lidocaine for menopausal dyspareunia in breast cancer survivors. ${ }^{9}$ Users of this solution reported less pain during sexual intercourse, decreased sexual distress, and improved sexual function.

Finally, Dr. Melisko turned briefly to problems with orgasm and low or lack of desire, libido, or intimacy. In the 2016 NCCN Guidelines, a discussion of options, including a vibrator or clitoral stimulatory device, with referral to an appropriate specialist is recommended. As for libido and desire issues, a discussion of available drugs, such as androgens and flibanserin, is warranted.
Approved by the FDA for premenopausal women in August 2015, flibanserin is not contraindicated in patients with breast cancer, although there are no clear data for this population. This approval is based on a meta-analysis of its use for hypoactive sexual desire disorder. ${ }^{10}$ Dr. Melisko admitted that for her, it is a controversial approval that requires future confirmatory studies. "This drug is not without side effects. It is taken every day, and in clinical trials side effects included substantial dizziness, somnolence, nausea, and fatigue."

\section{Treatment Alternatives for Men}

Dr. Narus reviewed some of the types of symptoms that may interfere with sexual function in male cancer survivors. These include erectile dysfunction, problems with ejaculation, difficulties with orgasm, and low or lack of desire, libido, or intimacy. Before 
discussing treatment options (Figure 2), Dr. Narus stressed the importance of motivation in patients and their spouses/partners when selecting the most appropriate option. The risks, benefits, and costs of treatment should be considered, and partner/spouse support is crucial to therapeutic success.

For erectile dysfunction, first-line treatment options in the 2016 NCCN Survivorship Guidelines for Sexual Function include lifestyle modifications for reversible causes, and oral agents such as phosphodiesterase type 5 (PDE5) inhibitors. "The introduction of sildenafil citrate in 1998 changed the management of erectile dysfunction," declared Dr. Narus. "Men now were able to have a noninvasive approach in the privacy of their own home." Again, Dr. Narus mentioned the importance of reviewing the risks and benefits and proper use of PDE5 inhibitors.

Dr. Narus noted that second-line options include vacuum erection devices, intracavernosal in- jection, and intraurethral suppositories. "All of these are well-studied options," he noted, "but they are not included in the NCCN Guidelines." A thirdline option is an implantable penile prosthesis; however, again, this option is not listed in the NCCN Guidelines. A referral to a sexual medicine clinician is recommended for second-and third-line therapies, according to Dr. Narus.

For ejaculation or orgasm disorders, the NCCN Guidelines recommend consideration of testosterone therapy if the total morning testosterone level is less than $300 \mathrm{ng} / \mathrm{dL}$; there is no contraindication for testosterone replacement therapy. For ejaculatory problems, psychological evaluation may be indicated. For orgasmic problems, treatment options listed include vibratory therapy, PDE5 inhibitors (if not contraindicated), and pelvic physical therapy. As for the symptoms of low or lack of desire, libido, or intimacy, the NCCN Guidelines recommend referral to a sexual health specialist. "It is important to

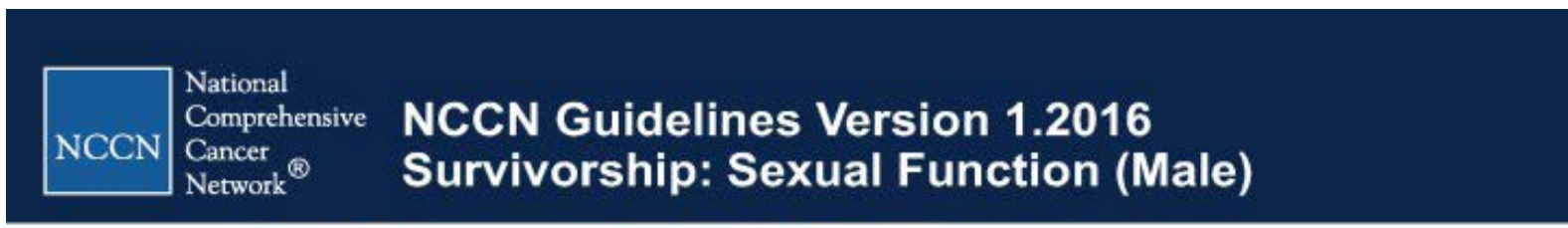

\section{SYMPTOMS}

TREATMENT OPTIONS

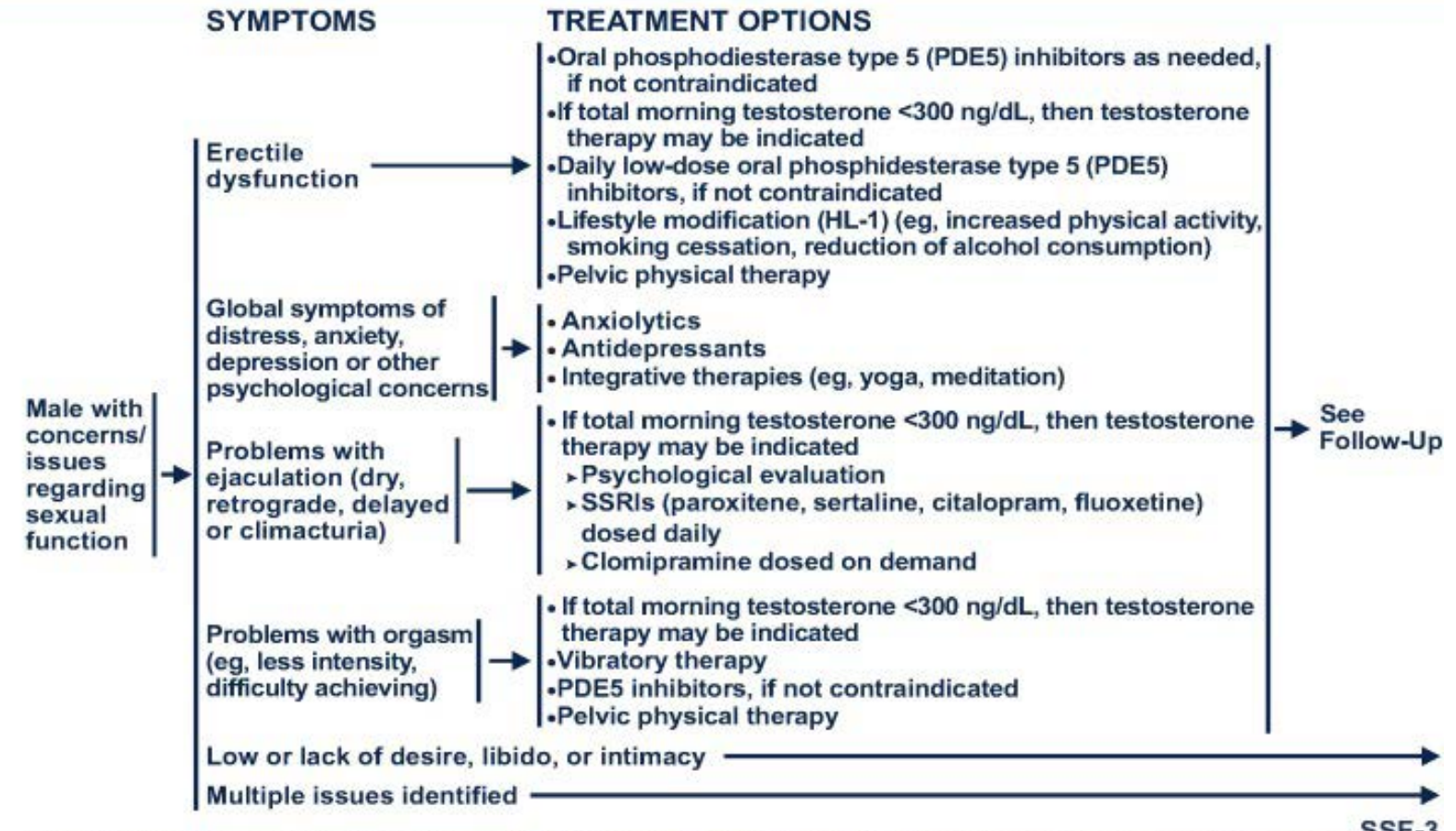

6 Nations Congreheneive Cancer Network, inc. 2016. All rights reserved. The NCCN Guidelines" and this iustration may not be reproduced in any form without the express written permission of NCCF" -3 
remember that not all treatments work for all men," stressed Dr. Narus.

At the close of his presentation, Dr. Narus made mention of some of the newer interventions that many men with sexual dysfunction may be considering, such as intercavernosal stem cell therapy, low-intensity shock wave therapy, and platelet-rich plasma therapy. ${ }^{11}$ However, these options are not FDA-approved, he cautioned, and there are no clinical data to support their use. "We want to use FDAapproved products on our patients," he concluded.

\section{References}

1. Jensen PT, Groenvold M, Klee MC, et al. Early-stage cervical carcinoma, radical hysterectomy, and sexual function: a longitudinal study. Cancer 2004;100:97-106.

2. Raggio GA, Butryn ML, Arigo D, et al. Prevalence and correlates of sexual morbidity in long-term breast cancer survivors. Psychol Health 2014;29:632-650.

3. Morales L, Neven P, Timmerman D, et al. Acute effects of tamoxifen and third-generation aromatase inhibitors on menopausal symptoms of breast cancer patients. Anticancer Drugs 2004;15:753-760.
4. Hendren SK, O'Connor BI, Liu M, et al. Prevalence of male and female sexual dysfunction is high following surgery for rectal cancer. Ann Surg 2005;242:212-223.

5. Cappelleri JC, Rosen RC. The Sexual Health Inventory for Men (SHIM): a 5-year review of research and clinical experience. Int J Impot Res 2005;17:307-319.

6. Rosen RC, Riley A, Wagner G, et al. The international index of erectile function: a multidimensional scale for assessment of erectile dysfunction. Urology 1997;49:822-830.

7. Mazzarello S, Hutton B, Ibrahim MF, et al. Management of urogenital atrophy in breast cancer patients: a systematic review of available evidence from randomized trials. Breast Cancer Res Treat 2015;152:1-8.

8. Portman D, Palacios S, Nappi RE, et al. Ospemifene, a non-oestrogen selective oestrogen receptor modulator for the treatment of vaginal dryness associated with postmenopausal vulvar and vaginal atrophy: a randomised, placebo-controlled, phase III trial. Maturitas 2014;78:91-98.

9. Goetsch MF, Lim JY, Caughey AB. A practical solution for dyspareunia in breast cancer survivors: a randomized controlled trial. J Clin Oncol 2015;33:3394-3400.

10. Jaspers L, Feys F, Bramer WM, et al. Efficacy and safety of flibanserin for the treatment of hypoactive sexual desire disorder in women: a systematic review and meta-analysis. JAMA Intern Med 2016;176:453-462.

11. Jenkins LC, Mulhall JP. The new business of ED therapy. J Sex Med 2015;12:2223-2225. 